\title{
CT evaluation of disease pattern in hilar cholangiocarcinoma
}

\begin{abstract}
Purpose: The objectives of our study were to map the various imaging patterns of hilar cholangiocarcinoma and identify commonest pattern.

Material and methods: In this retrospective study a search of the case records of the hospital using the key word cholangiocarcinoma in hospital information system and morphology code M-8160/3 yielded 288 patients of cholangiocarcinoma in the period April 2001 to June 2016. One hundred and fifty nine patients of intrahepatic and distal cholangiocarcinoma were excluded. Out of the remaining 129 patients with diagnosis of hilar cholangiocarcinoma, nine patients were excluded because available records were not complete. The remaining 120 patients formed the study sample.
\end{abstract}

Results: All the $120(100 \%)$ patients showed dilated intrahepatic biliary radicals. Periductal-infiltrating tumor was present in $117(97.50 \%)$ cases. According to BismuthCorlette classification on imaging, type I tumor was present in three $(02.50 \%)$, type II in $13(10.83 \%)$, type IIIa in $32(26.66 \%)$, type IIIb in $39(32.50 \%)$, and type IV in $33(27.50 \%)$ patients. The involvement of adjacent liver parenchyma was present in $60(50.00 \%)$ patients. Atrophy of a lobe was present in $75(62.50 \%)$ patients. Portal vein was involved in $91(75.83 \%)$ patients. These findings were common in massforming tumor.

Conclusion: Diagnosis of this rare entity can be done on imaging by identifying its typical pattern. In the majority cases tumor appears to be centered on the right or left hepatic duct with involvement of the ipsilateral portal vein, atrophy of hepatic lobe on that side and invasion of adjacent liver parenchyma. Periductal-infiltrating tumor was commonest morphological form and type IIIb was the commonest pattern.

Keywords: hilar cholangiocarcinoma, disease patterns, ct, biliary radicals, imaging, parenchyma, tumor, portal vein, morphological
Volume 2 Issue 2 - 2017

\author{
Mangal S Mahajan, Srikanth Moorthy, \\ Sreekumar P Karumathil, R Rajeshkannan, \\ Ramchandran Pothera \\ Department of Radiology, India
}

\begin{abstract}
Correspondence: Mangal S Mahajan, Department of Radiology, Bharati Vidyapeeth Medical College and Research Centre, Satara Road, Dhanakawadi, Pune - 4II 043, Maharashtra, India, Email drmangalmahajan@gmail.com
\end{abstract}

Received: October 21, 2016 | Published: February 22, 2017
Abbreviations: $\mathrm{CT}$, computed tomography; MDCT, multi-detector computed tomography; IHBRD, intrahepatic biliary radicals dilatation; MRCP, magnetic resonance cholangio pancreatography; $\mathrm{RPC}$, recurrent pyogenic cholangitis

\section{Introduction}

Incidence of cholangiocarcinoma varies worldwide. Most of studies of hilar cholangiocarcinoma are in the surgical literature ${ }^{1-4}$ and no large study has focused on the imaging aspects of the disease. This retrospective study mapped the various imaging patterns of hilar cholangiocarcinoma presenting to a tertiary referral center in a high incidence zone in southwestern India. Patients were imaged with single slice spiral CT and multiphase MDCT was used in the later part of the study.

Cholangiocarcinoma is the commonest primary bile duct cancer. It comprises of $10 \%-15 \%$ of hepatobiliary neoplasms. ${ }^{5}$ It is a rare disease accounting for $<2 \%$ of all human malignancies. ${ }^{6}$ Its cause is unknown and most cases are sporadic. There are numerous predisposing conditions that cause chronic biliary inflammation and subsequtly increase the risk of cholangiocarcinoma. They are primary sclerosing cholangitis, hepatobiliary flukes like Opisthorchis viverrini and Clonorchis sinensis, caroli disease, choledochal cyst, bile duct adenoma, biliary papillomatosis, chronic intraductal calculi, viral infections like human immunodeficiency virus, hepatitis B and $\mathrm{C}$ virus and Epstein-Barr virus, liver cirrhosis and chemicals like thorotrast, dioxin and vinyl chloride. ${ }^{6}$ It is classified as intrahepatic or extrahepatic; an intrahepatic cholangiocarcinoma is further classified as either peripheral or hilar. In the surgical literature, a tumor that arises peripheral to the secondary bifurcation of the left or right hepatic duct is considered to be peripheral cholangiocarcinoma. Extrahepatic tumor arises from hilar plate and common bile duct. A tumor that arises from right or left hepatic ducts or the bifurcation of the common hepatic duct is hilar cholangiocarcinoma(Klatskin tumor). ${ }^{7}$

Hilar cholangiocarcinoma was first described by Klatskin. ${ }^{4}$ It is categorized using Bismuth classification in to:

a) Type I: Tumors below the bifurcation of common hepatic duct;

b) Type II: Tumors involving the bifurcation; but not extending in to the main right and left duct;

c) Type III: Tumors infiltrating the right(IIIa) or the left(IIIb) hepatic duct;

d) Type IV: Tumors involving both the right and left hepatic duct (Figure 1). ${ }^{2}$

On the basis of classification scheme proposed by Japanese Liver Cancer Group, cholangiocarcinomas are classified into three types according to macroscopic appearance of the tumor: mass forming, intraductal growing, and periductal infiltrating; the last type is more prevalent in the hilar portion of the biliary tree. ${ }^{8,9}$ This classification is considered to be the most reasonable because it describes the 
gross appearance, growing characteristics and biologic behavior. It has prognostic implication for patients and helps radiologic interpretation. ${ }^{10}$ Cholangiocarcinoma is a slow-growing tumor which spreads along the bile ducts. Perineural and subepithelial extension is common. Regional lymph node involvement has been reported to occur in about $50 \%$ of patients while hematogenous metastasis is rare. Patients with advanced disease have a poor prognosis with very few surviving for more than one year. ${ }^{11}$

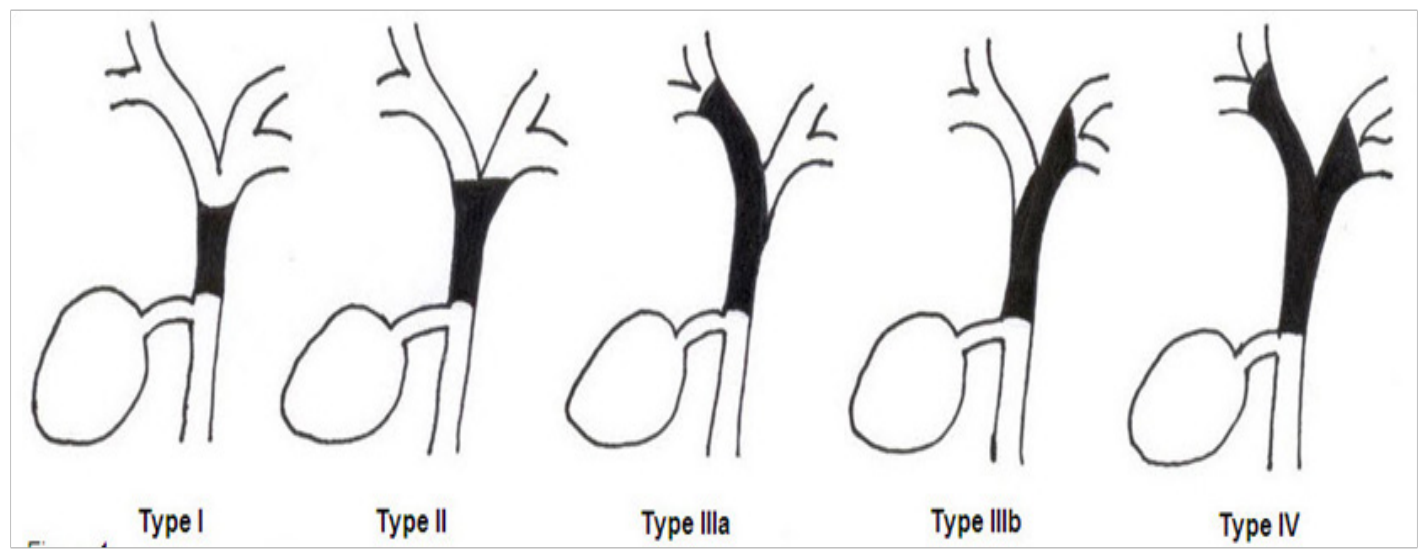

Figure I Schematic sketch of bismuth-corlette classification of ductal involvement in hilar cholangio carcinoma.

\section{Aims and objective}

A. To map the various imaging patterns of hilar cholangiocarcinoma.

B. To identify the commonest disease pattern.

\section{Materials and method}

Study design is descriptive. Case records of the hospital in the period April 2001 to June 2016 was searched using the key word cholangiocarcinoma in hospital information system and morphology code M-8160/3. Retrospective evaluation of case records of all patients with a diagnosis of hilar cholangiocarcinoma, which were radiologically and histologically proven was done. The clinical, imaging and histopathological records of the total 288 patients were evaluated. One hundred and fifty nine patients of intrahepatic and common bile duct cholangiocarcinomas were excluded. Of the remaining 129 patients of hilar cholangiocarcinoma four patients were excluded because available imaging study were not optimal and other five patients were excluded because histopathology was not available. Imaging of the remaining 120 patients which forms the study group was evaluated in detail. No informed consent was obtained from these patients as the study was retrospective. Following criteria was used to diagnose hilar cholangiocarcinoma: presence of enhancing periductal infiltrating or mass forming or intraductal growing mass at the hilum, presence of disproportionately dilated intrahepatic bile ducts with or without vascular and liver parenchymal invasion and atrophy-hypertrophy complex. Histologic confirmation of malignancy is not mandatory before exploration. In the absence of previous biliary tract surgery, the finding of focal stenotic lesion combined with the appropriate clinical presentation is sufficient for a presumptive diagnosis of hilar cholangiocarcinoma, which is correct in most instances. ${ }^{12}$

Out of 120 patients, scan was performed on 39 patients using single slice scanner(Somatom Plus 4, Siemens, Germany) before August 2007 and 81 patients were evaluated using 64 slice helical CT scanner(Sensation 64 slice CT, Siemens, Germany) after August 2007. All the patients were scanned using a standard protocol (Table 1). Delayed scan were obtained after 6 to 10 minutes in only 100 patients. The reason of not routinely obtaining the delayed scan was the difficulty in making a diagnosis of cholangiocarcinoma in all patients at the time of imaging.

All images were retrieved from picture archiving and communication system and viewed on a workstation. The presence of intrahepatic biliary dilatation, confluence involvement, morphology of mass, enhancement pattern, involvement of liver parenchyma and lobe involved in atrophy were recorded. Images were evaluated for vascular involvement of portal vein, hepatic artery, hepatic vein and inferior vena cava. Differential enhancement of the lobe on arterial phase in patients with portal vein invasion was also recorded. Involvement of liver parenchyma was considered when there is direct invasion of the liver tissue by tumor mass. The vessel was considered infiltrated if is stenosed, occluded, deformed due to adjacent tumor mass and/or more than 180 degree of its circumference was involved. Lobar atrophy was diagnosed when ductal crowding was present. Infiltration of liver parenchyma around the hilar mass was assessed. Presence of metastasis to the lymph node or distant organ was also recorded. Based on the above findings the imaging patterns were analyzed.

Interpretation of the images was done by two authors who had 9years and 21 years experience, respectively, in CT interpretation.

Table I Multi-detector computed tomography protocol used for scanning patients

\begin{tabular}{lll}
\hline Number & Parameter & Comment \\
\hline & & Plain scan - Domes of diaphragm to iliac crest. \\
& Arterial phase - Domes of diaphragm to iliac crest. \\
& Area scanned & Delayed phase - Domes of diaphragm to iliac crest. \\
& & Venous phase - Domes of diaphragm to ischial tuerosities. \\
& Scan direction & Craniocaudal \\
\hline
\end{tabular}




\begin{tabular}{|c|c|c|}
\hline Number & Parameter & Comment \\
\hline 3 & Peak voltage $\left(\mathrm{k} \mathrm{V}_{\mathrm{P}}\right)$ & 120 \\
\hline 4 & Tube current (mAs) & 230 \\
\hline 5 & Section thickness $(\mathrm{mm})$ & Arterial: 5, Venous: 8 , Delayed: 5 \\
\hline 6 & Reconstruction interval (mm) & Arterial: 5, Venous: 8, Delayed: 5 \\
\hline 7 & Table movement (mm/rotation) & $7.5 \mathrm{~mm}$ for arterial phase; $12 \mathrm{~mm}$ for venous phase and $7.5 \mathrm{~mm}$ for delayed phase. \\
\hline 8 & Pitch & 1.2 \\
\hline 9 & Rotation time (sec) & I \\
\hline \multirow[t]{4}{*}{10} & Contrast material injection & \\
\hline & a.Volume (ml) & $80 \mathrm{ml}+20 \mathrm{ml}$ saline. \\
\hline & b. Rate $(\mathrm{ml} / \mathrm{sec})$ & 2.3 \\
\hline & c. Scan delay (sec) & $\begin{array}{l}30 \mathrm{sec} \text { for arterial phase followed by venous phase at } 70 \mathrm{sec} \text { and delayed phase at } 6 \\
\text { to } 1 \text { minutes from the start of injection. }\end{array}$ \\
\hline II & Three dimensional technique & Multiplanar reconstruction. \\
\hline
\end{tabular}

$\mathrm{kV}$, kilovoltage peak; $\mathrm{mAs}$, milliamperage second; $\mathrm{mm}$, millimeter; sec, second; $\mathrm{ml}$, milliliter

\section{Results}

We studied 120 patients ranging between 41 to 98 years of age, out of which $75(62.50 \%)$ were males and $45(37.50 \%)$ were females. Overall mean age of presentation was $63.5 \pm 13.7$. For males it was $62.2 \pm 14.9$ and for females it was $65.7 \pm 11.3$. Majority of patients presented between 61 to 70 years of age.

All the 120 patients had total bilirubin level done. It was raised in 114 patients(95\%). Alkaline phosphatase was done in 120 cases and was elevated in all $(100 \%)$ the patients. All the studied patients showed dilated intrahepatic biliary radicals(100\%). Left lobe biliary radicals were dilated in all 120 patients. Right lobe showed IHBRD in 118 patients( $(98.33 \%)$.

Primary confluence was involved in $116(96.66 \%)$ patients and secondary confluence was involved in 104(86.66\%) patients. Right secondary confluence was isolated in 65 cases and left secondary confluence was isolated in 72 patients. On imaging, total number of patients according to Bismuth type are shown in Table $2 \&$ Figure $2-4$.

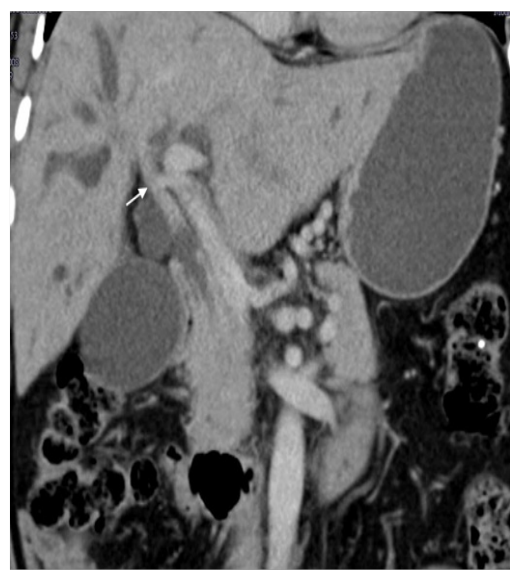

Figure $\mathbf{2}$ Coronal CT image of 68years old male shows periductal infiltrating type II hilar cholangiocarcinoma as concentric enhancing mass in common hepatic duct involving primary confluence (white arrow).

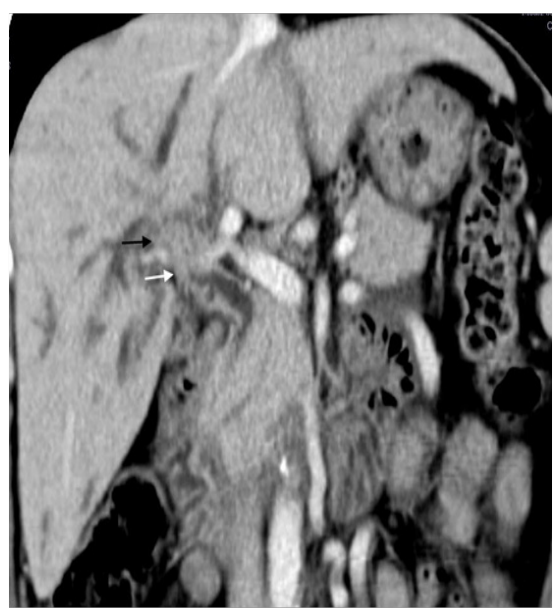

Figure 3 Type Illa well differentiated hilar cholangiocarcinoma treated with right hepatectomy - coronal CT image reveals tumor mass in common hepatic duct (white arrow) extending to right hepatic duct (black arrow).

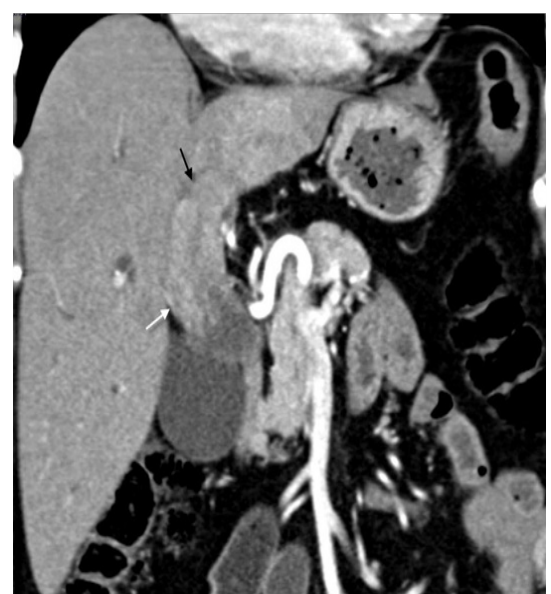

Figure 4 Type Illb intraductal-growing hilar cholangiocarcinoma-expansion of common (white arrow) and left hepatic duct (black arrow) due to lobulated intraductal growing tumor is well seen on coronal CT image. 
The tumor was categorized according to its morphological types in to mass-forming, periductal-infiltrating, and intraductal-growing (Table 3). Periductal-infiltrating tumor was the commonest form. Out of 120 , triple phase scan was performed in 100 patients. Out of 100 , only six $(6 \%)$ patients showed early enhancement of the tumor and in one patient the tumor was non-enhancing (1\%). 50 patient $(50 \%)$ showed enhancement of the tumor in portal phase and 43 patients (43\%) showed delayed enhancement of tumor. Only portal phase was performed in the remaining 20 patients and all showed enhancement of the tumor (Table 4).

Liver parenchymal involvement adjacent to the mass lesion was present in $60(50.00 \%)$ patients (Figure 5). Liver invasion was present in $39(41.48 \%)$ patients, and $21(91.30 \%)$ patients in periductal-infiltrating and mass-forming tumor respectively. None of the patient of intraductal-growing tumor showed liver invasion. Seventy five $(62.50 \%)$ patients had atrophy of the lobe, out of which $45(60.00 \%)$ patients had left lobe atrophy and $30(40.00 \%)$ patients had right lobe atrophy(either anterior or posterior segment or both). Lobar atrophy was present in one(33.33\%), three(23.07\%), 24(75.00\%), 29(74.35\%), and 18(54.54\%) patients in Bismuth-Corlette type I, II, IIIa, IIIb, and IV respectively (Figure 6). Lobar atrophy was present in $56(59.57 \%)$ patients, $18(78.26 \%)$ patients, and $1(3.33 \%)$ patient in periductal-infiltrating, mass-forming, and intraductal-growing tumor respectively.

Portal vein involvement was subcategorized as involvement of the main vein, left branch, right branch, and anterior and posterior division of right branch (Figure 5). In 91(75.83\%) patients, the portal vein was involved (Table 5A \& Table 5B). In 27 out of 32 patients of type IIIa, portal vein was involved. Ipsilateral(right side) branch involvement was seen in 24 cases whereas contralateral branch involvement was seen in two cases and main branch involvement was seen in one case without right branch invasion. In 32 out of 39 patients of type IIIb, left branch was involved and in two cases right branch was involved without left branch invasion. In type IV, invasion of main portal vein or it's right or left branch, or all three were seen in 24 patients. Two patients of type I and only four patients of type II showed portal venous invasion. Portal vein involvement was present in $70(74.46 \%)$ patients, $20(86.95 \%)$ patients, and $1(33.33 \%)$ patient in periductal-infiltrating, mass-forming, and intraductal-growing tumor respectively. Either main hepatic artery or its branches were involved in $57(47.50 \%)$ cases. Inferior vena cava was found to be involved in six $(05.00 \%)$ cases and hepatic veins were involved in $12(10.00 \%)$ cases. Out of 91 patients with portal vein involvement, differential enhancement of the lobes was seen in 44(48.35\%) patients (Figure 6).

Table 2 Division of patients according to bismuth-corlette classification

\begin{tabular}{lll}
\hline $\begin{array}{l}\text { Bismuth-corlette } \\
\text { classification }\end{array}$ & No. of patients $(\mathbf{n}=\mathbf{1 2 0})$ & Percentage \\
\hline I & 3 & $2.50 \%$ \\
II & 13 & $10.83 \%$ \\
IIIa & 32 & $26.66 \%$ \\
IIIb & 39 & $32.50 \%$ \\
IV & 33 & $27.50 \%$ \\
\hline
\end{tabular}

Table 3 Number of patients according to morphology of mass

\begin{tabular}{lll}
\hline $\begin{array}{l}\text { Morphology of mass } \\
\text { lesion }\end{array}$ & No. of patients $(\mathbf{n}=\mathbf{1 2 0})$ & Percentage \\
\hline Mass-forming & 23 & $19.16 \%$ \\
Periductal-infiltrating & 94 & $78.33 \%$ \\
Intraductal-growing & 3 & $2.50 \%$ \\
\hline
\end{tabular}

Table 4 Distribution of patients according to enhancement pattern

\begin{tabular}{lll}
\hline Enhancement pattern & No. of patients & Percentage \\
\hline No enhancement & 01 out of 100 & $1.00 \%$ \\
Early enhancement & 06 out of 100 & $6.00 \%$ \\
Portal enhancement & 70 out of 120 & $58.33 \%$ \\
Delayed enhancement & 44 out of 100 & $44.00 \%$ \\
\hline
\end{tabular}

Table 5A Patients with involvement of portal vein

\begin{tabular}{lll}
\hline $\begin{array}{l}\text { Portal vein } \\
\text { involvement }\end{array}$ & No. of Patients(n=120) & Percentage \\
\hline Present & 91 & $75.83 \%$ \\
Absent & 29 & $24.16 \%$ \\
\hline
\end{tabular}

Table 5B Distribution of patients with portal vein involvement

\begin{tabular}{lll}
\hline Portal vein involvement & $\begin{array}{l}\text { No. of } \\
\text { patients(n=9 I) }\end{array}$ & Percentage \\
\hline Main portal vein & 33 & $36.26 \%$ \\
$\begin{array}{l}\text { Left branch of portal vein } \\
\text { Right branch of portal vein }\end{array}$ & 65 & $71.42 \%$ \\
$\begin{array}{l}\text { Both main portal vein and its } \\
\text { left branch }\end{array}$ & 29 & $48.35 \%$ \\
$\begin{array}{l}\text { Both main portal vein and its } \\
\text { right branch }\end{array}$ & 24 & $31.86 \%$ \\
$\begin{array}{l}\text { All three (main portal vein, its } \\
\text { left and right branch) }\end{array}$ & 23 & $26.37 \%$ \\
\hline
\end{tabular}

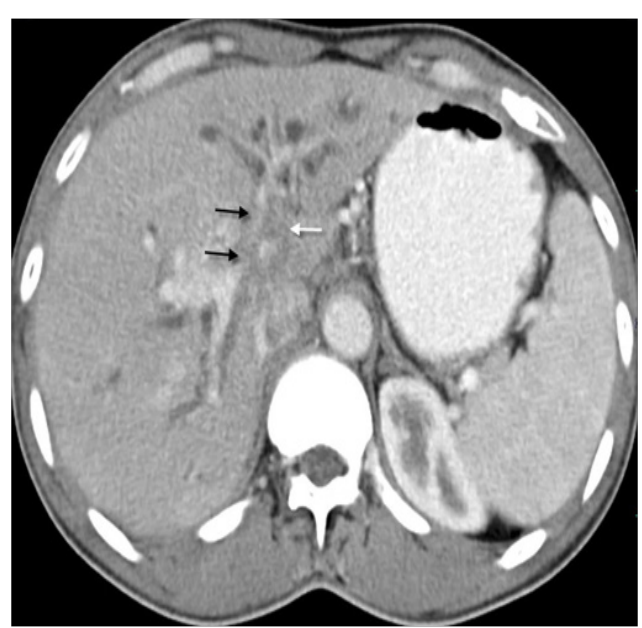

Figure 5 Type IV hilar cholangiocarcinoma - Infiltrating tumor (white arrow) involving the portal vein, its bifurcation and branches (black arrows) indicative of inoperable disease. 
Enlargement of lymph nodes in the pericystic duct, pericholedochal, hilar, periportal, periduodenal, peripancreatic, celiac, and superior mesenteric locations was assessed. In patients who underwent surgical resection, even lymph nodes less than $10 \mathrm{~mm}$ size were found to have metastatic deposit on histopathology. Hence we did not use size criteria for diagnosing nodal involvement. Any visible lymph node on imaging was documented. Distant metastasis was present in $18(15.00 \%)$ patients.

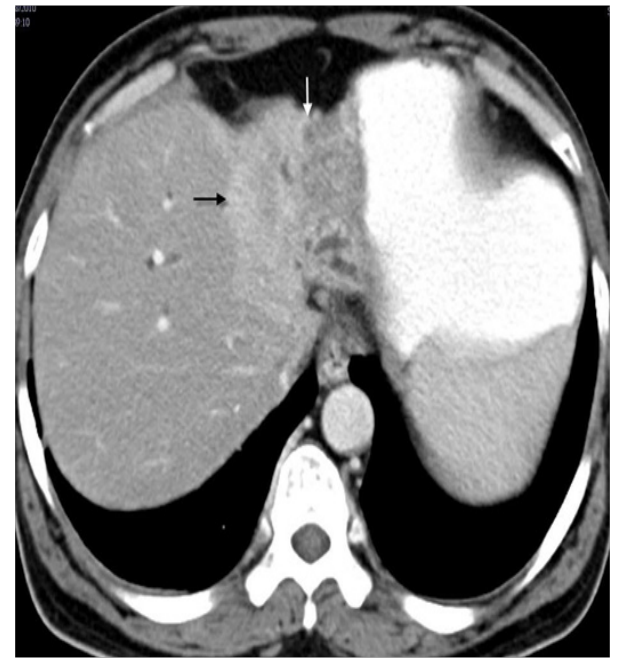

Figure 6 Atrophy (white arrow) and differential enhancement (black arrow) of the left lobe due to portal vein involvement in type Illb hilar cholangiocarcinoma.

\section{Discussion}

Cholangiocarcinoma is an adenocarcinoma that arises from the bile duct epithelium. ${ }^{13}$ It is more common in men, occurring commonly between the $6^{\text {th }}$ and $7^{\text {th }}$ decades. ${ }^{14}$ The prognosis has been considered worse for lesions affecting the confluence of the bile ducts and better for lesions close to the papilla. ${ }^{12}$ The yield of percutaneous needle biopsy or biliary brush cytology is poor and one may miss the opportunity to resects an early cancer. ${ }^{12}$ Hence the mainstay of preoperative diagnosis in hilar cholangiocarcinoma is imaging. To determine the operability and in order to select the appropriate surgical procedure, an accurate preoperative evaluation of both longitudinal spread and vertical invasion is a prerequisite..$^{15}$ Complete resection with or without partial hepatectomy and regional lymphadenectomy is the treatment of choice in selected patients. ${ }^{8}$ Therefore, imaging also plays an important role in determining whether a patient is a candidate for curative resection and in planning management. ${ }^{16}$ Helical CT and MRCP are the best imaging techniques to diagnose the disease, delineate the extent of the tumor and rule out liver metastasis and enlarged lymph nodes. ${ }^{17}$ MDCT allows for faster scanning with thinner collimation and can result in an improved diagnosis and evaluation of hilar cholangiocarcinoma. ${ }^{15}$ Our study aimed to map the different patterns of hilar cholangiocarcinoma and to identify the most common pattern of the disease. Knowledge of these patterns will help the radiologist to recognize the tumor early and look for findings which will impact therapeutic decision making. Most of the studies in the literature are surgical series which have not recorded the imaging findings in detail. The few series available on the imaging of hilar cholangiocarcinoma have included only small number of patients. Our retrospective analysis represents one of the largest series focusing on the imaging patterns of hilar cholangiocarcinoma.
Our patients ranged between 41 to 98 years. The mean age for males was $62.2 \pm 14.9$ years and for females $65.7 \pm 11$.3years. Majority of the patients presented in seventh decade. The age range was between 26-87years; mean age, 62years, in a study of Kim N R et al on 84 patients. ${ }^{18}$ Males to females ration in our study was $1.66: 1$. Poomphakwaen $\mathrm{K}$ et al. ${ }^{19}$ also recorded sex ratio of 2:1.

In patients with malignant biliary obstruction due to cholangiocarcinoma, the serum bilirubin level often achieves levels greater than $10 \mathrm{mg} / \mathrm{dl}$ and averages $18 \mathrm{mg} / \mathrm{dl}$, whereas patients with obstruction from choledocholithiasis have lower bilirubin levels. ${ }^{12}$ One hundred and fourteen patients in our series showed elevated total bilirubin level. Out of these, 90 patients had level more than $10 \mathrm{mg} / \mathrm{dl}$.

Periductal-infiltrating tumor was the most common morphological type found in our study. This was followed by mass-forming tumor and intraductal-growing tumor. Longlin $\mathrm{Y}$ et al..$^{20,21}$ in their study also found that the periductal-infiltrating tumor was commonest and intraductal-growing umor was rarest morphological forms.

Early enhancement was present in only six patients out of 100 in our series whereas a study by Chryssou et al. ${ }^{21}$ observed arterial enhancement in four of their 26 patients. Seventy patients out of 120 showed enhancement in portal phase. Of the total hundred cases in which delayed scans were performed, retention of contrast was seen in forty three cases. Takayasu $\mathrm{K}$ et al. ${ }^{22}$ in their study found that delayed enhancement was present in all patients of hilar cholangiocarcinoma. Chryssou et al. ${ }^{21}$ also observed delayed enhancement of the tumor in $84.61 \%$ of the patient. The variability in the observed incidence of delayed enhancement in the literature may be related to the different delay times employed by the investigators for delayed scans and variation in the sample size.

Invasion of adjacent liver parenchyma is important in determining tumor respectability. ${ }^{23}$ Our study revealed that involvement of liver parenchyma adjacent to mass lesion was present in $50 \%$ patients. In a study of Manfredi $\mathrm{R}$ et al. ${ }^{23} \mathrm{MR}$ imaging studies accurately showed local parenchymal invasion in nine $(75 \%)$ of 12 patients and underestimated in three $(25 \%)$ of 12 patients. Liver parenchymal invasion was common in mass-forming tumors in our study. Exact incidence of the liver invasion according to the morphological type of the tumor is not mentioned in the literature to the best of our knowledge.

Segmental or lobar atrophy may result from a portal venous occlusion or biliary obstruction. One or both of these findings are often present in patients with hilar cholangiocarcinoma. Long standing biliary obstruction itself can cause parenchymal atrophy. When portal vein branch is also obstructed, the atrophy becomes severe. Identification of lobar atrophy at imaging has implications for management. ${ }^{12}$ No liver resection should be performed that leaves an atrophic remnant. ${ }^{23}$ Our series showed atrophy in $75(62.50 \%)$ cases. It was commonly observed in mass-forming tumors. In a study by Feydy A et al. ${ }^{24}$ on eleven patients, helical CT showed lobar atrophy in $\operatorname{six}(54.54 \%)$ patients. We observed more frequent left lobe atrophy $(60 \%)$ than right lobe involvement $(40 \%)$ (either anterior or posterior segment or both). None of the patients had atrophy of both right and left lobes. The reason for preferential involvement of left lobe is unclear. Exact incidence of lobar atrophy according to the morphological type of the tumor is not mentioned in the literature to the best of our knowledge.

Portal venous involvement is a distinctive feature of 
cholangiocarcinoma in late stages.$^{25}$ Amongst the three morphological types, portal vein involvement was frequently present in mass-forming tumor. Sasaki A et al in their study of 37 patients also showed common involvement of portal vein in mass-forming tumor. ${ }^{26}$ This hilar neoplasm can involve main portal vein or its right or left branches. Majority of the patients in our study showed ipsilateral involvement of portal vein branch in type IIIa and IIIb $(75.00 \%$ in type IIIa and $82.05 \%$ in type IIIb). Atrophy of the lobe was commonest on the side of involvement of secondary confluence and portal vein branch. This pattern suggests that, the tumor may originate in the left or the right hepatic duct and then spreads to involve the ipsilateral secondary confluence and portal vein branch, then the primary confluence, the common hepatic duct and finally the opposite side secondary confluence. Hyperperfusion of the lobe on arterial phase on the side of portal vein branch involvement was also a common feature.

The study was retrospective and included only patients with a diagnosis of hilar cholangiocarcinoma. It was not possible to derive the sensitivity and specificity of the imaging criteria to diagnose the disease prospectively. Both MDCT and MRCP were not performed in all the patients of the study group, hence comparison between these modalities were not made. Moreover the performance of a test changes according to prevalence of a disease. It is possible that a typical imaging pattern described here would warrant consideration of alternative diagnosis also when encountered in a low incidence area.

\section{Differential diagnosis}

Many neoplastic and non-neoplastic diseases can be misdiagnosed as hilar cholangiocarcinoma on imaging. Common differentials of the hilar cholangiocarcinoma are gall bladder carcinoma, Mirrizi syndrome, primary sclerosing cholangitis, and RPC. Uncommon conditions includes inflammatory pseudotumor of the bile duct, intrabiliary metastasis, benign biliary tumors, lymphoma and carcinoids of the bile duct. Dilated biliary radical is common in infiltrating gall bladder carcinoma which commonly presents as a mass replacing the gall bladder. The tumor infiltrates along the cystic duct to the extrahepatic bile duct and this intraductal spread of the tumor results in biliary obstruction and dilatation. ${ }^{27}$ Mirrizi syndrome consists of common hepatic duct obstruction due to compression by the stone impacted in the gall bladder neck or cystic duct. In type I syndrome inflammation around common bile duct leads to stricture formation and thus mimics periductal-infiltrating type of cholangiocarcinoma. In type II syndrome there is cholecysto-biliary fistula. ${ }^{28}$ Primary sclerosing cholangitis is characterized by diffuse cholangitis and progressive fibrosis of both intrahepatic and extrahepatic bile ducts. Common findings are multifocal strictures, duct wall thickening and irregular beaded appearance of involved ducts. Rarely, focal segmental involvement is difficult to distinguish from mimic's periductal-infiltrating type of cholangiocarcinoma. Recurrent pyogenic cholangitis is characterized by repeated attacks of acute pyogenic cholangitis on the background of biliary obstruction by pigmented calculi or biliary strictures. Segmental distribution in RPC has predilection for lateral segment of left lobe, posterior segment of right lobe and the extrahepatic bile ducts. ${ }^{29}$ Inflammatory pseudotumor is rare condition that often resembles aggressive malignancy. Imaging features of this benign entity are nonspecific and indistinguishable from those of mass-forming type of cholangiocarcinoma. A repeated negative frozen section margin is the helpful indicator of benign disease..$^{29,30}$ Intrabiliary metastasis is very rare. Common tumors that metastasize to bile ducts are cancers of lung, breast, gall bladder, colon, testis, prostate, pancreas and me- lanomas. Lymphoma of the bile duct is rare and is usually secondary to systemic disease..$^{29,31}$

\section{Conclusion}

Periductal-infiltrating tumor was most common morphological form. Liver parenchymal invasion, lobar atrophy, and portal vein involvement was frequently observed in mass-forming tumor. Liver parenchymal invasion, portal vein involvement, lobar atrophy and hyperperfusion of the lobe on the side of the involved hepatic duct were frequent finding in type III tumor. These findings were uncommon in type I and type II tumors. The commonest pattern of hilar cholangiocarcinoma in our series was involvement of left secondary confluence with invasion of ipsilateral portal vein, adjacent liver parenchymal infiltration, and lobar atrophy. Awareness of these common findings will aid the prospective imaging diagnosis this rare disease.

\section{Acknowledgements}

None.

\section{Conflict of interest}

Author declares that there is no conflict of interest.

\section{References}

1. Wei-Liang Y, Xin-Chen Z, Dong-Wei Z, et al. Diagnosis and surgical treatment of hepatic hilar cholangiocarcinoma. Hepatobiliary Pancreat Dis Int. 2007;6:631-635.

2. Mansfield SD, Barakat O, Charnley RM, et al. Management of hilar cholangiocarcinoma in the North of England: Pathology, treatment and outcome. World J Gastroenterol. 2005;11(48):7625-7630.

3. Kobayashi A, Miwa S, Nakata T, et al. Disease recurrence patterns after R0 resection of hilar cholangiocarcinoma. Br J Surg. 2010;97(1):56-64.

4. Papoulas M, Lubezky N, Goykhman Y, et al. Contemporary surgical approach to hilar cholangiocarcinoma. Isr Med Assoc J. 2011;13(2):99-103.

5. Lazaridis KN, Gores GJ. Cholangiocarcinoma. Gastroenterology 2005;128(6):1655-1667.

6. Slattery JM, Sahani DV. What is the current state-of-the-art imaging for detection and staging of cholangiocarcinoma? The Oncologist. 2006;11(8):913-922.

7. Han FK, Choi BI, Kim AY, et al. Cholangiocarcinoma: Pictorial essay of CT and cholangiographic findings. Radio Graphics. 2002;22(1):173-187.

8. Lee HY, Kim SH, Lee JM, et al. Preoperative assessment of resectability of hepatic hilar cholangiocarcinoma: Combined CT and cholangiography with revised criteria. Radiology. 2006;239(1):113-121.

9. Chung YE, Kim MF, Pak YN, et al. Varying Appearances of Cholangiocarcinoma: Radiologic-Pathologic Correlation. RadioGraphics. 2009;29(3):683-700.

10. Lim JH. Cholangiocarcinoma: Morphologic classification according to growth pattern and imaging findings. AJR. 2003;181(3):819-827.

11. Khan SA, Davidson BR, Goldin R, et al. Guidelines for the diagnosis and treatment of cholangiocarcinoma: consensus document. Gut. 2002;51(6):1-9.

12. Jarnagin WR, Angelica MD, Blumgart LH. Intrahepatic and extrahepatic biliary cancers. Blumgart: Surgery of the liver, biliary tract, and pancreas. 4th ed. Philadelphia: Saunders Elsevier; 2007. p. 1747-1759. 
13. Vilgrain V. Staging cholangiocarcinoma by imaging studies. $H P B$ 2008;10(2):106-109.

14. Vanderveen KA, Hussain HK. Magnetic resonance imaging of cholangiocarcinoma. Cancer Imaging. 2004;4(2):104-115.

15. Akamatsu N, Sugawara Y, Osada H, et al. Diagnostic accuracy of multidetector-row computed tomography for hilar cholangiocarcinoma. $J$ Gastroenterol Hepatol. 2010;25(4):731-737.

16. Choi JY, Kim MJ, Lee JM, et al. Hilar cholangiocarcinoma: Role of preoperative imaging with sonography, MDCT, MRI, and direct cholangiography. AJR Am J Roentgenol. 2008;191(5):1448-1457.

17. Figueras J, Llado L, Valls C, et al. Changing strategies in diagnosis and management of hilar cholangiocarcinoma. Liver Transpl. 2000;6(6):786-794

18. Kim NR, Lee JM, Kim SH, et al. Enhancement characteristics of cholangiocarcinoma on multiphase helical CT: emphasis on morphological subtypes. Clin Imaging. 2008;32:114-120.

19. Poomphakwaen K, Promthet S, Kamsa-ard S, et al. Risk factors for cholangiocarcinoma in Khon Kaen, Thailand: A nested case-control study. Asian Pac J Cancer Prev. 2009;10(2):251-258.

20. Longlin Y, Song B, Xu J, et al. Hilar Cholangiocarcinoma: Diagnosis and Evaluation of Resectability with the Three-Dimensional Thin-section Contrast-enhanced Dynamic MR Imaging Sequence. International Journal of Magnetic Resonance Imaging. 2007;1(1):33-42.

21. Chryssou E, Guthrie JA, Ward J, et al. Hilar cholangiocarcinoma: MR correlation with surgical and histological findings. Clin Rodiol. 2010;65(10):781-788

22. Takayasu K, Ikeya S, Mukai K, et al. CT of Hilar Cholangiocarcinoma: Late Contrast Enhancement in Six Patients. AJR. 1990;154(6):1203-1206.
23. Manfredi R, Masselli G, Maresca G, et al. MR imaging and MRCP of hilar cholangiocarcinoma. Abdom Imaging. 2003;28(3):319-325.

24. Feydy A, Vilgrain V, Denys A, et al. Helical CT assessment in hilar cholangiocarcinoma: Correlation with surgical and pathological findings. AJR. 1999;172(1):73-77.

25. Mahajan MS, Moorthy S, Karumathil SP, et al. Hilar Cholangiocarcinoma: Cross Sectional Evaluation of Disease Spectrum. IJRI. 2015;25(2):184-192.

26. Sasaki A, Aramaki M, Kawano AK, et al. Intrahepatic peripheral cholangiocarcinoma: mode of spread and choice of surgical treatment. $\mathrm{Br} \mathrm{J}$ Surg. 1998;85(9):1206-1209.

27. Levi AD, Murakata LA, Roharmann CA. Gall bladder carcinoma: Radiologic-Pathologic correlation. Radiographics. 2001;21(2):295-314.

28. Valls C, Ruiz S, Martinez L, et al. Radiological diagnosis and staging of hilar cholangiocarcinoma. World $J$ Gastrointest Oncol. 2013;5(7):115-126.

29. Menias CO, Surabhi VR, Prasad SR, et al. Mimics of cholangiocarcinoma: Spectrum of disease. Radiographics. 2008;28(4):1115-1129.

30. Tublin ME, Moser AJ, Marsh JW, et al. Biliary inflammatory pseudotumor: Imaging features in seven patients. AJR Am J Roentgenol. 2007; 188(1):44-48.

31. Ashkar L, Maheshwari S, Pressacco J, et al. MR Cholangiopancreatography. Clinical Magnetic Resonance Imaging. 3rd ed. Philadelphia: Saunders Elsevier; 2005. p. 4695-4711. 